\title{
Fuzzy Semi-Numbers and Their Elementary Arithmetic With a Medical Case Study
}

\author{
Sh. Yeganehmanesh ${ }^{1}$ M. Amirfakhrian ${ }^{1}$, P. Grzegorzewski ${ }^{2,3}$ \\ ${ }^{1}$ Department of Mathematics, Central Tehran Branch, Islamic Azad University, \\ Tehran, Iran. \\ E-mail: shahab.yeganehmanesh@gmail.com,amirfakhrian@iauctb.ac.ir \\ ${ }^{2}$ Systems Research Institute, Polish Academy of Sciences, \\ Newelska 6, 01-447, Warsaw, Poland. \\ ${ }^{3}$ Faculty of Mathematics and Information Science, Warsaw University of Technology, \\ Koszykowa 75, 00-662, Warsaw, Poland. \\ E-mail: pgrzeg@ibspan.waw.pl \\ Received प0ए XQ 2010 \\ Accepted 10R\$ SUO2010
}

\begin{abstract}
A new methodology for processing non-normal fuzzy sets is proposed. To break the predominant constraint on normality of fuzzy numbers the concept of fuzzy semi-numbers is introduced Then it is shown how to generalize operations defined on fuzzy numbers onto a family of fuzzy semi-numbers with possibly different heights.
\end{abstract}

Keywords: Fuzzy Number, Fuzzy Arithmetic, Fuzzy Semi-Number, Elevated Fuzzy Semi-Number.

\section{Introduction}

In most of the papers on fuzzy sets the authors consider only fuzzy numbers, i.e. fuzzy sets which are convex and normal (a fuzzy set is normal if its height is one, i.e. its membership function reaches value 1 at least in one point of its domain). Moreover, elementary arithmetic has been defined just on fuzzy numbers ${ }^{22,28}$ and, consequently, most of the contributions devoted to fuzzy ranking ${ }^{1,7,36}$, fuzzy approximation ${ }^{2,3,4,8,13,16,37}$, fuzzy differential equations ${ }^{18,27}$, fuzzy integral equations 21,31 and systems of fuzzy equations ${ }^{11,33}$ are restricted to fuzzy numbers, especially to trapezoidal fuzzy numbers $1,9,24,25$. Fuzzy sets with height less than one ap-

\footnotetext{
* Corresponding author.
}

pear have appeared in the literature rarely ${ }^{26,32}$. In particular, Chen considered the so-called Generalized Fuzzy Numbers (GFN) ${ }^{15}$, i.e. fuzzy sets with triangular and trapezoidal membership functions but with height less than one.

To mitigate the possible information loss caused by applying normalization $14,35,41$ and minimum height methods ${ }^{10,12}$, we propose a new methodology for processing non-normal fuzzy sets. To eliminate the predominant constraint on normality we introduce the novel concept of fuzzy semi-numbers and suggest how to generalize operations defined on fuzzy numbers onto the broad family of fuzzy seminumbers irrespective on their heigth.

The structure of this paper is as follows. In Sec- 
tion 2 we recall basic concepts related to fuzzy numbers. Next, in Section 3, we introduce fuzzy seminumbers and define basic arithmetic operations on fuzzy semi-numbers of the same height, which further on are called equally high fuzzy semi-numbers. Then, in Section 4, we consider functions on equally high fuzzy semi-numbers. In Section 5 we introduce two useful operators: the elevator and fuzzy elevator which appear very helpful in generalizing arithmetic operations on fuzzy semi-numbers with arbitrary height. This way, in Section 6, we introduce a new method for performing calculations on arbitrary fuzzy semi-numbers. In Section 7 we provide a motivational medical case study showing some advantages of the proposed methodology. Finally, Section 8 concludes the contribution.

\section{Preliminaries}

Before delving into our contribution, let's take a glance at some basic fuzzy theory definitions.

Let $\widetilde{u}$ denote a fuzzy set defined on the real line $\mathbb{R}$ with its membership function $\mu_{\widetilde{u}}: \mathbb{R} \rightarrow[0,1]$.

Definition 2.1. The support of a fuzzy set $\widetilde{u}$ is defined as follows ${ }^{20}$ :

$$
\operatorname{supp}(\widetilde{u})=\left\{x \mid \mu_{\widetilde{u}}(x)>0\right\} .
$$

Definition 2.2. The $\alpha$-cut (where $\alpha \in[0,1]$ ) of a fuzzy set $\widetilde{u}$ is defined by ${ }^{41}$ :

$$
[\widetilde{u}]^{\alpha}= \begin{cases}\left\{x \mid \mu_{\widetilde{u}}(x) \geqslant \alpha\right\} & \text { if } \alpha \in(0,1], \\ \operatorname{supp}(\widetilde{u}) & \text { if } \alpha=0 .\end{cases}
$$

Definition 2.3. A fuzzy set $\widetilde{u}$ is convex if $\forall x, y \in \mathbb{R}$ and $\forall \lambda \in[0,1]$ we have ${ }^{41}$ :

$$
\mu_{\widetilde{u}}(\lambda x+(1-\lambda) y) \geqslant \min \left\{\mu_{\widetilde{u}}(x), \mu_{\widetilde{u}}(y)\right\}
$$

Accordingly, if all $\alpha$-cuts of $\widetilde{u}$ are convex, then $\widetilde{u}$ is a convex fuzzy set.

Definition 2.4. The hight of a fuzzy set $\widetilde{u}$ is given by convex if $\forall x, y \in \widetilde{S}$ and $\forall \lambda \in[0,1]$ we have ${ }^{41}$ :

$$
\operatorname{hgt}(\widetilde{u})=\sup _{x \in \mathbb{R}} \mu_{\widetilde{u}}(x) .
$$

Definition 2.5. A fuzzy set $\widetilde{u}$ is called normal if given by $\operatorname{hgt}(\widetilde{u})=1$.
Definition 2.6. The core of a normal fuzzy set $\widetilde{u}$ is defined as follows ${ }^{20}$ :

$$
\operatorname{core}(\widetilde{u})=\left\{x \mid \mu_{\widetilde{u}}(x)=1\right\} .
$$

Let $F(\mathbb{R})$ be the set of all fuzzy numbers, (i.e. the set of all normal and convex fuzzy sets ${ }^{17,41}$ ) on the real line.

Definition 2.7. A generalized $L R$ fuzzy number $\widetilde{u}$ with the membership function $\mu_{\widetilde{u}}(x), x \in \mathbb{R}$ can be defined as $2,3,4,37$ :

$$
\mu_{\widetilde{u}}(x)= \begin{cases}l_{\widetilde{u}}(x), & a \leqslant x \leqslant b, \\ 1, & b \leqslant x \leqslant c, \\ r_{\widetilde{u}}(x), & c \leqslant x \leqslant d, \\ 0, & \text { otherwise }\end{cases}
$$

where $l_{\widetilde{u}}$ is the left membership function and $r_{\widetilde{u}}$ is the right membership function. It is assumed that $l_{\widetilde{u}}$ is increasing in $[a, b]$ and $r_{\tilde{u}}$ is decreasing in $[c, d]$, and that $l_{\widetilde{u}}(a)=r_{\widetilde{u}}(d)=0$ and $l_{\widetilde{u}}(b)=r_{\widetilde{u}}(c)=1$. In addition, if $l_{\widetilde{u}}$ and $r_{\widetilde{u}}$ are linear, then $\widetilde{u}$ is a trapezoidal fuzzy number, which is denoted by $\widetilde{u}=(a, b, c, d)$. Moreover, if $b=c$, we have a so-called triangular fuzzy number and we denote it by $\widetilde{u}=(a, c, d)$.

We say that a fuzzy number $\widetilde{u}$ is presented in its parametric form if $\widetilde{u}=(\underline{u}, \bar{u})$, where $\underline{u}$ and $\bar{u}$ denote the left and right spread functions, respectively. Functions $\underline{u}(\alpha)$ and $\bar{u}(\alpha)$ over $[0,1]$, satisfy the following requirements $6,29,30$ :

1. $\underline{u}$ is increasing and left continuous,

2. $\bar{u}$ is decreasing and left continuous,

3. $\underline{u}(\alpha) \leqslant \bar{u}(\alpha), \quad 0 \leqslant \alpha \leqslant 1$.

Actually, given a family of $\alpha$-cuts of a fuzzy number $\widetilde{u}$, i.e. $[\widetilde{u}]^{\alpha}$ for $\alpha \in[0,1]$, defined by (2.1), we obtain

$$
\begin{aligned}
& \underline{u}=\inf [\widetilde{u}]^{\alpha}, \\
& \bar{u}=\sup [\widetilde{u}]^{\alpha} .
\end{aligned}
$$

Note, that a crisp number $a$ is a singleton set $\{a\}$ with height 1 . Hence $\underline{u}(\alpha)=\bar{u}(\alpha)=a, \forall \alpha \in[0,1]$, and we may denote it as $a=\{a\}$.

Let $\widetilde{u}=(\underline{u}, \bar{u}), \widetilde{v}=(\underline{v}, \bar{v}) \in F(\mathbb{R})$ and $k \in \mathbb{R}$. Basic arithmetic operations on fuzzy numbers $\widetilde{u}$ and $\widetilde{v}$ (i.e. addition, subtraction and product by scalar) are defined as follows ${ }^{19,28}$ : 
- $k>0: k \widetilde{u}=(k \underline{u}, k \bar{u})$;

- $k<0: k \widetilde{u}=(k \bar{u}, k \underline{u})$;

- $\widetilde{u}+\widetilde{v}=(\underline{u}+\underline{v}, \bar{u}+\bar{v})$;

- $\widetilde{u}-\widetilde{v}=(\underline{u}-\bar{v}, \bar{u}-\underline{v})$.

A fuzzy number $\tilde{u}$ is non-negative (non-positive) if for $x<0(x>0)$ we have $\mu_{\widetilde{u}}(x)=0$. Similarly, a fuzzy number $\widetilde{v}$ is positive (negative) if $\mu_{\tilde{v}}(x)=0$ for $x \leqslant 0(x \geqslant 0)$.

Definition 2.8. A fuzzy set with membership function $\mu$ is called a Generalized Fuzzy Number ${ }^{15}$ if the following conditions hold:

(i) $\mu$ is a continuous mapping from $\mathbb{R}$ to the closed interval $[0, h], 0<h \leqslant 1$,

(ii) $\mu(x)=0$ for $x \in(-\infty, a]$,

(iii) $\mu$ is strictly increasing on $[a, b]$,

(iv) $\mu(x)=h$ for $x \in[b, c]$,

(v) $\mu$ is strictly decreasing on $[c, d]$,

(vi) $\mu(x)=0$ for $x \in[d,+\infty)$.

\section{Fuzzy Semi-Numbers}

Here we present several novel definitions and lemmas which will be used throughout the paper.

Definition 3.1. A fuzzy set $\widetilde{u}_{h}$ is a generalized fuzzy $L R$ semi-number ${ }^{5,38}$, if there exist a positive number $h \in(0,1]$ such that

$$
\mu_{\widetilde{u}_{h}}(x)=\left\{\begin{array}{cc}
l(x), & a \leqslant x \leqslant b, \\
h, & b \leqslant x \leqslant c, \\
r(x), & c \leqslant x \leqslant d, \\
0, & \text { otherwise }
\end{array}\right.
$$

where $l(x)$ is nondecreasing on $[a, b]$ and $r(x)$ is nonincreasing on $[c, d]$ such that $l(a)=r(d)=0$ and $l(b)=r(c)=h$.

We denote a fuzzy $L R$ semi-number by $(a, b, c, d ; h)_{L R}$. Obviously, if $h=1$ then $\widetilde{u}$ is an $L R$ fuzzy number.

A fuzzy semi-number is a (possibly) non-normal fuzzy set with the aforementioned conditions. It is clear that the set of all fuzzy semi-numbers is a subset of a family of all fuzzy sets and a superset of the family of of fuzzy numbers. Figure 1 shows a nonnormal fuzzy set which is not a fuzzy semi-number because for lack of convexity .

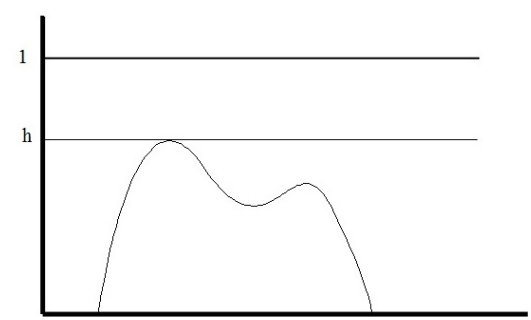

Fig. 1. A non-normal fuzzy set which is not a fuzzy seminumber.

The membership function of GFNs should be strictly increasing (decreasing) on left (right) spread but in fuzzy semi-numbers it should be only nondecreasing (nonincreasing). Therefore, the set of fuzzy semi-numbers is a superset of the family of the generalized fuzzy numbers. Figure 2 shows a fuzzy semi-number which is not a GFN because of the aforementioned reasons.

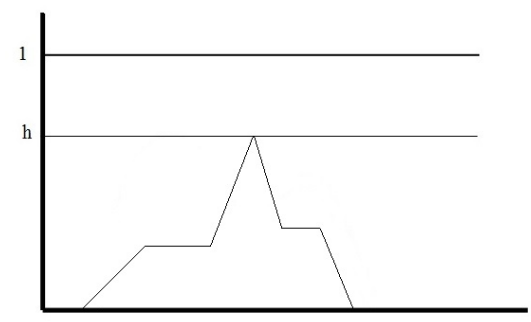

Fig. 2. A fuzzy semi-number which is not a GFN.

Moreover, a membership function of a GFN should be continuous, which is not required $i$ the case of fuzzy semi-numbers. Figure 3 illustrates a fuzzy semi-number which is not a GFN.

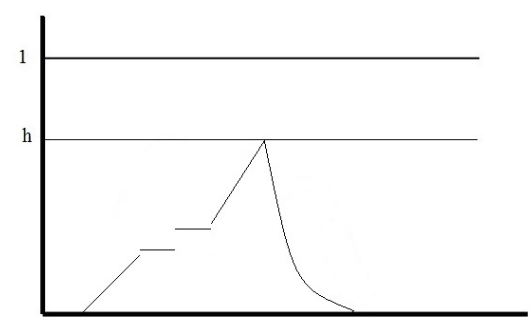

Fig. 3. A fuzzy semi-number which is not a GFN. 
Definition 3.2. Fuzzy semi-numbers $\widetilde{u}$ and $\widetilde{v}$ are called equally high if they have the same height, i.e.hgt $(\widetilde{u})=\operatorname{hgt}(\widetilde{v})$.

We denote the set of all fuzzy semi-numbers of height $h$ by $F_{h}(\mathbb{R})$. Therefore, the set of all fuzzy semi-numbers, denoted by $F S(\mathbb{R})$, satisfies

$$
F S(\mathbb{R})=\bigcup_{h \in(0,1]} F_{h}(\mathbb{R}) .
$$

It is clear that $F(\mathbb{R})$ is a proper subset of $F S(\mathbb{R})$.

If $l(x)$ and $r(x)$ are linear, then $\widetilde{u}_{h}$ is a trapezoidal fuzzy semi-number, which is denoted by $(a, b, c, d ; h)$. Moreover, if $b=c$, we obtain a triangular fuzzy semi-number and denote it by $(a, b, d ; h)$. Let $T F(\mathbb{R})=\{(a, b, c, d): a \leqslant b \leqslant$ $c \leqslant d\}$ and $T F_{h}(\mathbb{R})=\{(a, b, c, d ; h): a \leqslant b \leqslant c \leqslant$ $d, 0<h \leqslant 1\}$ denote the set of all trapezoidal fuzzy numbers and the set of all trapezoidal equally high fuzzy semi-numbers with height $h$, respectively. It $T F S(\mathbb{R})$ denote the set of all trapezoidal fuzzy seminumbers then

$$
T F S(\mathbb{R})=\bigcup_{h \in(0,1]} T F_{h}(\mathbb{R}) .
$$

Definition 3.3. Suppose $\widetilde{u}_{h} \in F_{h}(\mathbb{R})$. Then the $\mathrm{H}$-core of a fuzzy semi-number $\widetilde{u}_{h}$ is defined as follows ${ }^{38}$ :

$$
\mathrm{H} \text {-core }\left(\widetilde{u}_{h}\right)=\left\{x \mid \mu_{\widetilde{u}}(x)=h\right\} .
$$

For any fuzzy semi-number $\widetilde{u}_{h} \in F_{h}(\mathbb{R})$ we can consider its $\alpha$-cuts defined by (2.1) for $\alpha \in[0, h]$. Similarly, by (2.2)-(2.3) we define functions $\underline{u}(\alpha)$ and $\bar{u}(\alpha)$ for $\alpha \in[0, h]$. This way we obtain the parametric form of a fuzzy semi-number given by $\widetilde{u}_{h}=(\underline{u}, \bar{u} ; h)$, where functions $\underline{u}$ and $\bar{u}$ satisfy the following requirements:

1. $\underline{u}$ is increasing and left continuous on $[0, h]$.

2. $\bar{u}$ is decreasing and left continuous on $[0, h]$.

3. $\underline{u}(\alpha) \leqslant \bar{u}(\alpha), \quad 0 \leqslant \alpha \leqslant h$.

In particular, for a trapezoidal fuzzy seminumber $\widetilde{u}_{h}=(a, b, c, d ; h)$ we obtain

$$
\begin{aligned}
& \underline{u}(\alpha)=a+\frac{b-a}{h} \alpha, \\
& \bar{u}(\alpha)=d-\frac{d-c}{h} \alpha,
\end{aligned}
$$

where $0 \leqslant \alpha \leqslant h$.

Since a fuzzy semi-number with height $h=1$ is a fuzzy number, hence further on in such cases we'll omit height in the notation, i.e. we denote $\widetilde{u}_{1}=(\underline{u}, \bar{u} ; 1)$ by $\widetilde{u}=(\underline{u}, \bar{u})$.

A crisp semi-number ${ }^{38} a_{h}^{\circ}$ is a fuzzy singleton set with height $h$. Then $\underline{u}(\alpha)=\bar{u}(\alpha)=a, \forall \alpha \in[0, h]$, and we denote it as $a_{h}^{\circ}=\{a ; h\}$. We denote the set of all crisp semi-numbers of height $h$ by $\mathbb{R}_{h}^{\circ}$. Hence $\mathbb{R}_{1}^{\circ}=\mathbb{R}$.

\section{Functions on equally high fuzzy semi-numbers}

Suppose we want to generalize a mapping $f$ : $\mathbb{R}^{n} \rightarrow \mathbb{R}$ onto the framework of fuzzy semi-numbers. Thus we are interested in a mapping from $F_{h}^{n}(\mathbb{R})$ into $F_{h}(\mathbb{R})$, where $F_{h}^{n}(\mathbb{R})=\left\{\widetilde{\mathbf{u}}_{h}=\left(\widetilde{u}_{1, h}, \ldots, \widetilde{u}_{n, h}\right)\right.$ : $\left.\widetilde{u}_{1, h}, \ldots, \widetilde{u}_{n, h} \in F_{h}(\mathbb{R})\right\}$, such that

$$
\widetilde{v}_{h}=f\left(\widetilde{\mathbf{u}}_{h}\right) .
$$

By the Extension Principle, the membership function of $\widetilde{v}_{h} \in F_{h}(\mathbb{R})$ is given by

$\mu_{\widetilde{v}}(y)=\left\{\begin{array}{lr}\sup _{\left(x_{1}, \ldots, x_{n}\right) \in f^{-1}(y)} \min \left\{\boldsymbol{\mu}_{\widetilde{u}_{1, h}}\left(x_{1}\right), \ldots, \mu_{\widetilde{u}_{n, h}}\left(x_{n}\right)\right\} \\ 0, & \text { if } f^{-1}(y) \neq \emptyset, \\ 0, & \text { otherwise. }\end{array}\right.$

In particular, if $f: F_{h}(\mathbb{R}) \rightarrow F_{h}(\mathbb{R})$ is a univariate function then

$$
\mu_{\widetilde{v}_{h}}(y)=\sup _{y=f(x)} \mu_{\widetilde{u}_{h}}(x)
$$

which for an invertible function $f$ reduces to

$$
\mu_{\widetilde{v}_{h}}(y)=\mu_{\widetilde{u}_{h}}\left(f^{-1}(y)\right) .
$$

One can easily notice that for $f: \mathbb{R} \rightarrow \mathbb{R}$ and a fuzzy semi-number $\widetilde{u}_{h}=(\underline{u}, \bar{u} ; h)$ in $F_{h}(\mathbb{R})$ we obtain

$$
f\left(\widetilde{u}_{h}\right)=(f(\underline{u}), f(\bar{u}) ; h) .
$$

The proof of the following useful lemma is straightforward. 
Lemma 4.1. Let $\left(\widetilde{u}_{h}, \widetilde{v}_{h}\right)$ be a pair of fuzzy seminumbers in $F_{h}^{2}(\mathbb{R})$ and let $f: \mathbb{R}^{2} \rightarrow \mathbb{R}$ be an increasing function with respect to both variables. Then, it is:

$$
f\left(\widetilde{u}_{h}, \widetilde{v}_{h}\right)=(f(\underline{u}, \underline{v}), f(\bar{u}, \bar{v}) ; h) .
$$

Due to the aforementioned considerations we can define basic arithmetic operations on equally high fuzzy semi-numbers.

Definition 4.1. Let $\widetilde{u}_{h}=(\underline{u}, \bar{u} ; h)$ and $\widetilde{v}_{h}=(\underline{v}, \bar{v} ; h)$ be two equally high fuzzy semi-numbers with height $h \in(0,1]$. Then

$$
\begin{aligned}
\widetilde{u}_{h}+\widetilde{v}_{h} & =(\underline{u}+\underline{v}, \bar{u}+\bar{v} ; h), \\
\widetilde{u}_{h} \cdot \widetilde{v}_{h} & =(\underline{u \cdot v}, \overline{u \cdot v} ; h),
\end{aligned}
$$

where

$$
\begin{aligned}
& \underline{u \cdot v}=\min \{\underline{u} \cdot \underline{v}, \underline{u} \cdot \bar{v}, \bar{u} \cdot \underline{v}, \bar{u} \cdot \bar{v}\}, \\
& \overline{u \cdot v}=\max \{\underline{u} \cdot \underline{v}, \underline{u} \cdot \bar{v}, \bar{u} \cdot \underline{v}, \bar{u} \cdot \bar{v}\} .
\end{aligned}
$$

Lemma 4.2. If both of $\widetilde{u}_{h}$ and $\widetilde{v}_{h}$ are two nonpositive or non-negative fuzzy semi-numbers belonging to $F_{h}(\mathbb{R})$, then

$$
\widetilde{u}_{h} \cdot \widetilde{v}_{h}=(\underline{u} \cdot \underline{v}, \bar{u} \cdot \bar{v} ; h) .
$$

Proof. We know that $(x, y) \rightarrow x y$ is an increasing function over $\mathscr{A}^{2}$ and $\mathscr{B}^{2}$ where $\mathscr{A}=[0,+\infty)$ and $\mathscr{B}=(-\infty, 0]$. Therefore, according to Lemma 4.1 the proof is completed.

Lemma 4.3. If $\widetilde{u}_{h}$ is a non-negative fuzzy seminumber and $\widetilde{v}_{h}$ is a non-positive fuzzy semi-number belonging to $F_{h}(\mathbb{R})$, then

$$
\widetilde{u}_{h} \cdot \widetilde{v}_{h}=(\underline{u} \cdot \bar{v}, \bar{u} \cdot \underline{v} ; h) .
$$

And if $\widetilde{u}_{h}$ is a non-positive fuzzy semi-number and $\widetilde{v}_{h}$ is a non-negative fuzzy semi-number belonging to $F_{h}(\mathbb{R})$, then

$$
\widetilde{u}_{h} \cdot \widetilde{v}_{h}=(\bar{u} \cdot \underline{v}, \underline{u} \cdot \bar{v} ; h) .
$$

Proof. We know that $(x, y) \rightarrow x y$ is a decreasing function over $\mathscr{A} \times \mathscr{B}$ where $\mathscr{A}=[0,+\infty)$ and $\mathscr{B}=(-\infty, 0]$. And similarly, we know that $(x, y) \rightarrow x y$ is a decreasing function over $\mathscr{B} \times \mathscr{A}$ where $\mathscr{A}=[0,+\infty)$ and $\mathscr{B}=(-\infty, 0]$. Thus, according to Lemma 4.1 the proof is completed.

Note, that $0_{h}^{\circ}=(0 ; h)$ is the additive identity in $F_{h}(\mathbb{R})$, while $1_{h}^{\circ}=(1 ; h)$ is the multiplicative identity in $F_{h}(\mathbb{R})$. Indeed

$$
\widetilde{u}_{h}+0_{h}^{\circ}=(\underline{u}+0, \bar{u}+0 ; h)=(\underline{u}, \bar{u} ; h)=\widetilde{u}_{h}
$$

and

$$
\widetilde{u}_{h} \cdot 1_{h}^{\circ}=(\underline{u \cdot 1}, \overline{u \cdot 1} ; h)=(\underline{u}, \bar{u} ; h)=\widetilde{u}_{h} .
$$

We can also define the scalar multiplication, subtraction ad division in $F_{h}(\mathbb{R})$.

Definition 4.2. Let $\widetilde{u}_{h}=(\underline{u}, \bar{u} ; h) \in F_{h}(\mathbb{R})$ and $k \in$ $\mathbb{R}_{h}^{\circ}$. Then

$$
k \widetilde{u}_{h}= \begin{cases}(k \underline{u}, k \bar{u} ; h) & \text { if } k_{h}^{\circ}>0_{h}^{\circ}, \\ (k \bar{u}, k \underline{u} ; h) & \text { if } k_{h}^{\circ}<0_{h}^{\circ},\end{cases}
$$

Definition 4.3. Let $\widetilde{u}_{h}=(\underline{u}, \bar{u} ; h)$ and $\widetilde{v}_{h}=(\underline{v}, \bar{v} ; h)$ be two equally high fuzzy semi-numbers with height $h \in(0,1]$. Then

$$
\begin{aligned}
& \widetilde{u}_{h}-\widetilde{v}_{h}=(\underline{u}-\bar{v}, \bar{u}-\underline{v} ; h), \\
& \widetilde{u}_{h} \div \widetilde{v}_{h}=\left(\left(\frac{u}{v}\right), \bar{u}\left(\frac{u}{v}\right) ; h\right),
\end{aligned}
$$

where

$$
\begin{aligned}
& \frac{\left(\frac{u}{v}\right)}{\overline{\bar{u}}}=\min \{\underline{u} / \underline{v}, \underline{u} / \bar{v}, \bar{u} / \underline{v}, \bar{u} / \bar{v}\}, \\
& \left(\frac{\left.\frac{v}{v}\right)}{}=\max \{\underline{u} / \underline{v}, \underline{u} / \bar{v}, \bar{u} / \underline{v}, \bar{u} / \bar{v}\} .\right.
\end{aligned}
$$

Let $a_{h}^{\circ}, b_{h}^{\circ} \in \mathbb{R}_{h}^{\circ}$ be two equally high crisp seminumbers. The proof of the following facts is straightforward:

$$
\begin{aligned}
a_{h}^{\circ}+b_{h}^{\circ} & =(a+b)_{h}^{\circ}, \\
a_{h}^{\circ}-b_{h}^{\circ} & =(a-b)_{h}^{\circ}, \\
a_{h}^{\circ} \cdot b_{h}^{\circ} & =(a b)_{h}^{\circ}, \\
\frac{a_{h}^{\circ}}{b_{h}^{\circ}} & =\left(\frac{a}{b}\right)_{h}^{\circ}, \quad b \neq 0 .
\end{aligned}
$$

Let us consider the following examples. 
Example 4.1. Let $\widetilde{u}_{\frac{1}{2}}$ be a fuzzy semi-number with height $\frac{1}{2}$ given by the following membership function

$$
\mu_{\widetilde{u}_{\frac{1}{2}}}(x)= \begin{cases}\frac{x-1}{2}, & 1 \leqslant x<2 \\ \frac{5-x}{6}, & 2 \leqslant x<5 .\end{cases}
$$

Let $\widetilde{v}_{\frac{1}{2}}=f\left(\widetilde{u}_{\frac{1}{2}}\right)=\exp \left(\widetilde{u}_{\frac{1}{2}}\right)$ be a function on $F_{h}(\mathbb{R})$ to itself. Since $f$ is a monotonic function hence by (4.2) we obtain $\mu_{\widetilde{v}_{\frac{1}{2}}}(y)=\mu_{\widetilde{u}_{\frac{1}{2}}}\left(f^{-1}(y)\right)=\mu_{\widetilde{u}_{\frac{1}{2}}}(\ln (y))$ given by

$$
\mu_{\widetilde{v}_{\frac{1}{2}}}(y)= \begin{cases}\frac{\ln (y)-1}{2}, & e \leqslant y<e^{2}, \\ \frac{5-\ln (y)}{6}, & e^{2} \leqslant y<e^{5} .\end{cases}
$$

Membership functions of $\widetilde{u}_{\frac{1}{2}}$ and $\widetilde{v}_{\frac{1}{2}}$ are given in Figures 4 and 5 , respectively.

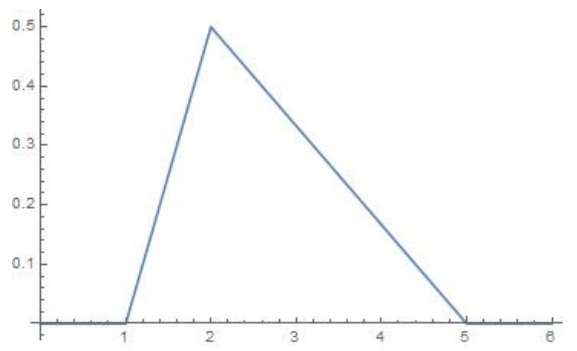

Fig. 4. A membership function of $\widetilde{u}_{\frac{1}{2}}$ in Ex. 4.1.

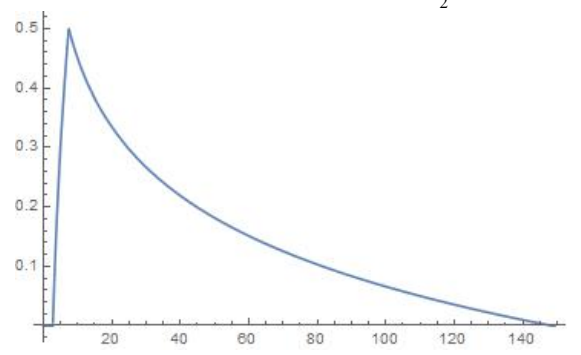

Fig. 5. A membership function of $\exp \left(\widetilde{u}_{\frac{1}{2}}\right)$ in Ex. 4.1.

Let us also consider $\widetilde{w}_{\frac{1}{2}}=g\left(\widetilde{u}_{\frac{1}{2}}\right)=\ln \widetilde{u}_{\frac{1}{2}}$. Since $g$ is a monotonic function, we obtain $\mu_{\widetilde{w}_{\frac{1}{2}}}(y)=$ $\mu_{\widetilde{u}_{\frac{1}{2}}}\left(f^{-1}(y)\right)=\mu_{\widetilde{u}_{\frac{1}{2}}}(\exp (y))$, where

$$
\mu_{\widetilde{w}_{\frac{1}{2}}}(y)= \begin{cases}\frac{\exp (y)-1}{2}, & 0 \leqslant y<\ln 2 ; \\ \frac{5-\exp (y)}{6}, & \ln 2 \leqslant y<\ln 5 .\end{cases}
$$

The membership functions of $\widetilde{w}_{\frac{1}{2}}$ is given in Figure 6.

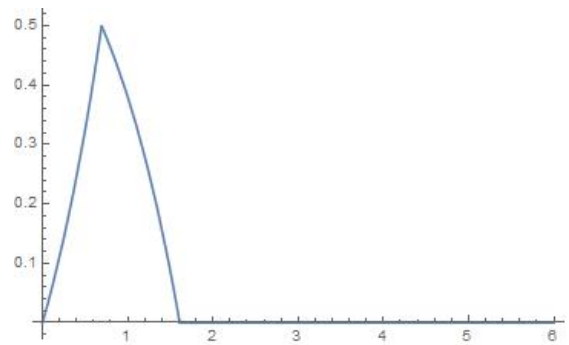

Fig. 6. A membership function of $\ln \left(\widetilde{u}_{\frac{1}{2}}\right)$ in Ex. 4.1.

Example 4.2. Let $\widetilde{u}_{\frac{1}{3}}$ be a fuzzy semi-number with height $\frac{1}{3}$ with the following membership function:

$$
\mu_{\widetilde{u}_{\frac{1}{3}}}(x)=\left\{\begin{array}{lc}
\frac{2}{\pi} x, & 0 \leqslant x<\frac{\pi}{6}, \\
\frac{\pi}{2}-x & \frac{\pi}{\pi} \leqslant x<\frac{\pi}{2} .
\end{array}\right.
$$

Let $\widetilde{v}_{\frac{1}{3}}=k\left(\widetilde{u}_{\frac{1}{3}}\right)=\sin \left(\widetilde{u}_{\frac{1}{3}}\right)$. Since $k$ is an one to one function in $\left[0, \frac{\pi}{2}\right]$ hence we have $\mu_{\widetilde{v}_{\frac{1}{3}}}(y)=$ $\mu_{\widetilde{u}_{\frac{1}{3}}}\left(k^{-1}(y)\right)=\mu_{\widetilde{u}_{\frac{1}{3}}}(\arcsin (y))$

$$
\begin{aligned}
\mu_{\widetilde{v}_{\frac{1}{3}}}(y) & = \begin{cases}\frac{2}{\pi} \arcsin (y), & 0 \leqslant \arcsin (y)<\frac{\pi}{6}, \\
\frac{\frac{\pi}{2}-\arcsin (y)}{\pi}, & \frac{\pi}{6} \leqslant \arcsin (y)<\frac{\pi}{2}\end{cases} \\
& = \begin{cases}\frac{2}{\pi} \arcsin (y), & 0 \leqslant y<\frac{1}{2}, \\
\frac{\frac{\pi}{2}-\arcsin (y)}{\pi}, & \frac{1}{2} \leqslant y<1 .\end{cases}
\end{aligned}
$$

Membership functions of $\widetilde{u}_{\frac{1}{2}}$ and $\widetilde{v}_{\frac{1}{2}}$ are given in Figures 7 and 8 , respectively. 


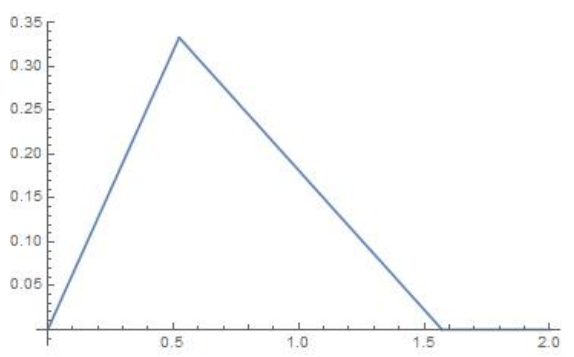

Fig. 7. A membership function of $\widetilde{u}_{\frac{1}{3}}$ in Ex. 4.2.

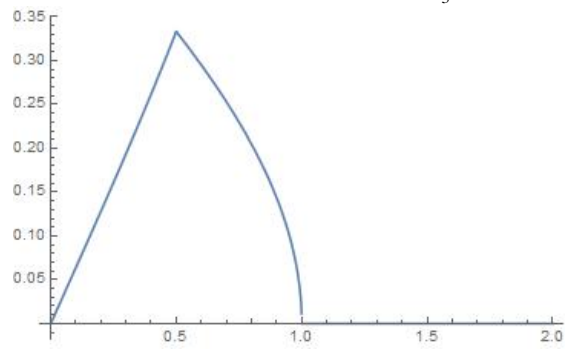

Fig. 8. A membership function of $\sin \left(\widetilde{u}_{\frac{1}{3}}\right)$ in Ex. 4.2.

\section{Fuzzy elevator operator}

In this section we introduce two operators: elevator and fuzzy elevator, which will come in handy in presentation of elementary arithmetic operations on fuzzy semi-numbers in general form.

Definition 5.1. Let $C_{M L}(A)$ be the set of all monotonic left continuous functions over the set $A$. Let $f(\alpha) \in C_{M L}([0, h])$, where $h \leqslant 1$. An elevator operator is a mapping $E_{h}^{h^{*}}: C_{M L}([0, h]) \longrightarrow C_{M L}\left(\left[0, h^{*}\right]\right)$, where $h^{*} \in[h, 1]$, such that $f^{*}=E_{h}^{h^{*}}(f)$, where $f^{*}$ is given as follows ${ }^{5}$ :

$$
f^{*}(\alpha)=\left\{\begin{array}{lc}
f(\alpha), & \alpha \in(0, h], \\
f(h), & \alpha \in\left(h, h^{*}\right] \\
0, & \alpha \notin\left(0, h^{*}\right]
\end{array}\right.
$$

It is clear that $f^{*}$ is an extension of $f$, i.e. $\left.f^{*}\right|_{(0, h]}(\alpha)=f(\alpha)$ for any $\alpha \in(0, h]$. It is also easily seen that an elevator is a linear operator, i.e. for any two monotonic left continuous functions $f$ and $g$ and a real number $k$ we have

$$
E_{h}^{h^{*}}(k f+g)=k E_{h}^{h^{*}}(f)+E_{h}^{h^{*}}(g)
$$

Definition 5.2. A fuzzy elevator is an operator $E_{h}^{h^{*}}$ : $F_{h}(\mathbb{R}) \longrightarrow F_{h^{*}}(\mathbb{R})$ defined as follows ${ }^{5}$ :

$$
\begin{aligned}
\widetilde{E}_{h}^{h^{*}}\left(\widetilde{u}_{h}\right) & =\widetilde{E}_{h}^{h^{*}}(\underline{u}, \bar{u} ; h) \\
& =\left(E_{h}^{h^{*}}(\underline{u}), E_{h}^{h^{*}}(\bar{u}) ; h^{*}\right) \\
& =\left(\underline{u}^{*}, \bar{u}^{*} ; h^{*}\right)=\widetilde{u}_{h^{*}}^{*},
\end{aligned}
$$

where $\widetilde{u}_{h}=(\underline{u}, \bar{u} ; h) \in F_{h}(\mathbb{R})$ and $h^{*} \in[h, 1]$. A fuzzy semi-number $\widetilde{u}_{h^{*}}^{*}=\left(\underline{u}^{*}, \bar{u}^{*} ; h^{*}\right) \in F_{h^{*}}(\mathbb{R})$ is called the elevated fuzzy semi-number.

By Definition 5.1 functions $\underline{u}^{*}(\alpha)$ and $\bar{u}^{*}(\alpha)$ describing the elevated fuzzy semi-number $\widetilde{u}_{h^{*}}^{*}=$ $\left(\underline{u}^{*}, \bar{u}^{*} ; h^{*}\right)$ are obtained by applying an elevator operator to $\underline{u}$ and $\bar{u}$, respectively ${ }^{5}$. Hence we obtain

$$
\underline{u}^{*}(\alpha)=\left\{\begin{array}{lc}
\underline{u}(\alpha), & \alpha \in(0, h], \\
\underline{u}(h), & \alpha \in\left(h, h^{*}\right], \\
0, & \alpha \notin\left(0, h^{*}\right]
\end{array}\right.
$$

and

$$
\bar{u}^{*}(\alpha)=\left\{\begin{array}{lc}
\bar{u}(\alpha), & \alpha \in(0, h], \\
\bar{u}(h), & \alpha \in\left(h, h^{*}\right], \\
0, & \alpha \notin\left(0, h^{*}\right] .
\end{array}\right.
$$

We say that fuzzy semi-numbers $\widetilde{u}_{h}=(\underline{u}, \bar{u} ; h) \in$ $F_{h}(\mathbb{R})$ and $\widetilde{u}_{h^{*}}^{*}=\left(\underline{u}^{*}, \bar{u}^{*} ; h^{*}\right) \in F_{h^{*}}(\mathbb{R})$ are associated.

The proofs of the following lemmas are straightforward.

Lemma 5.1. Let $\widetilde{u}_{h}=(\underline{u}, \bar{u} ; h) \in F_{h}(\mathbb{R})$ be an arbitrary fuzzy semi-number. The the spread functions $\underline{u}^{*}(\alpha)$ and $\bar{u}^{*}(\alpha)$ of its associated elevated fuzzy semi-number $\widetilde{u}_{h^{*}}^{*}=\left(\underline{u}^{*}, \bar{u}^{*} ; h^{*}\right) \in F_{h^{*}}(\mathbb{R})$ satisfy the following properties:

1. $\underline{u}^{*}$ is an increasing left continuous function over $\left[0, h^{*}\right]$,

2. $\bar{u}^{*}$ is a decreasing left continuous function over $\left[0, h^{*}\right]$,

3. $\underline{u}^{*}(\alpha) \leqslant \bar{u}^{*}(\alpha), \quad 0 \leqslant \alpha \leqslant h^{*}$,

4. $\underline{u}^{*}(\alpha)=\bar{u}^{*}(\alpha)=0, \quad \alpha \notin\left(0, h^{*}\right]$.

Lemma 5.2. Fuzzy semi-numbers are closed under fuzzy elevator, i.e. the elevated fuzzy semi-number with respect to a given height $h^{*}$ is a fuzzy seminumber with height $h^{*}$.

Obviously, the elevated of a crisp semi-number $a_{h}^{\circ}$ with respect to a given height $h^{*}$ is a crisp seminumber $a_{h^{*}}^{\circ}$. 
Example 5.1. Figures 9 and 10 show the spread functions of a fuzzy semi-number $\widetilde{u}=\left(1,2,3,5 ; \frac{1}{3}\right)$ and its associated elevated fuzzy semi-number with respect to height $\frac{2}{3}$.

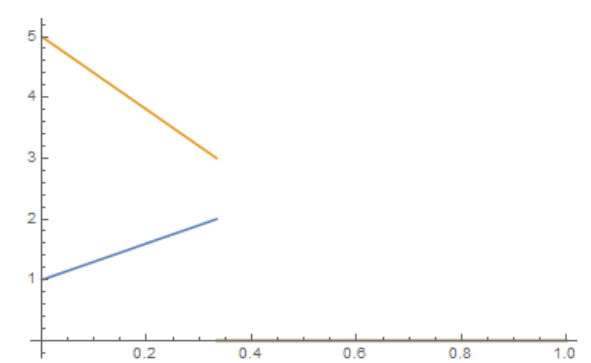

Fig. 9. The spread functions of a fuzzy semi-number $\widetilde{u}=$ $\left(1,2,3,5 ; \frac{1}{3}\right)$.

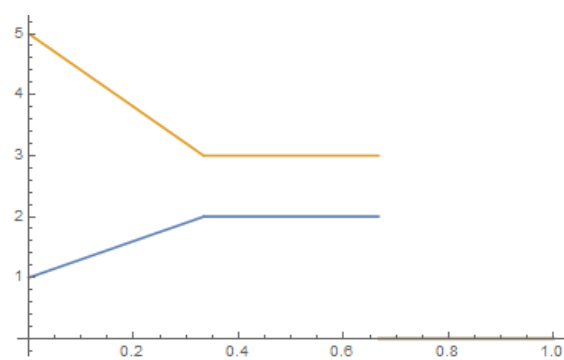

Fig. 10. The spread functions of the elevated form of $\widetilde{u}=\left(1,2,3,5 ; \frac{1}{3}\right)$ with respect to height $\frac{2}{3}$.

Similarly, Figures 11 and 12 present the spread functions of a fuzzy semi-number $\widetilde{u}=\left(1,2,2,5 ; \frac{1}{3}\right)$ and its associated elevated fuzzy semi-number with respect to height $\frac{2}{3}$

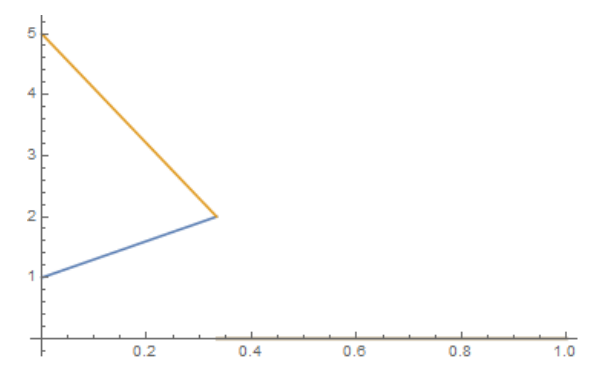

Fig. 11. The spread functions of $\widetilde{u}=\left(1,2,2,5 ; \frac{1}{3}\right)$.

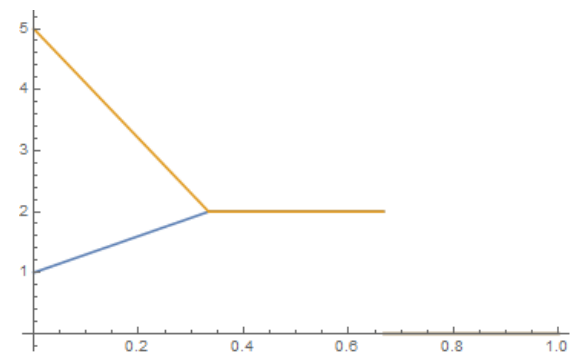

Fig. 12. The spread functions of the elevated form of $\widetilde{u}=\left(1,2,2,5 ; \frac{1}{3}\right)$ with respect to height $\frac{2}{3}$.

\section{Arithmetic operations on general fuzzy semi-numbers}

To perform fuzzy arithmetic on non-normal fuzzy sets two main approaches have been used so far. Firstly, one may normalize the non-normal fuzzy sets by dividing them by their heights and then apply the desired operations well-defined for fuzzy numbers ${ }^{14,35,41}$. Alternatively, one may define an operation by the extension principle with respect to the minimum heights of non-normal fuzzy sets ${ }^{10,12}$. In this section, we propose another method for defining elementary arithmetic operations on fuzzy seminumber with different heights utilizing heir elevated forms with respect to the maximum of their heights. Definition 6.1. Let $\widetilde{u}_{h_{u}}=\left(\underline{u}, \bar{u} ; h_{u}\right)$ and $\widetilde{v}_{h_{v}}=$ $\left(\underline{v}, \bar{v} ; h_{v}\right)$ be any fuzzy semi-numbers and let $h^{*}=$ $\max \left\{h_{u}, h_{v}\right\}$. Then basic arithmetic operations on fuzzy semi-numbers are defined as follows:

- addition

$$
\widetilde{u}_{h_{u}}+\widetilde{v}_{h_{v}}=\widetilde{u}_{h^{*}}^{*}+\widetilde{v}_{h^{*}}^{*}=\left(\underline{u}^{*}+\underline{v}^{*}, \bar{u}^{*}+\bar{v}^{*} ; h^{*}\right),
$$

- subtraction

$$
\widetilde{u}_{h_{u}}-\widetilde{v}_{h_{v}}=\widetilde{u}_{h^{*}}^{*}-\widetilde{v}_{h^{*}}^{*}=\left(\underline{u}^{*}-\bar{v}^{*}, \bar{u}^{*}-\underline{v}^{*} ; h^{*}\right) .
$$

- multiplication

$$
\widetilde{u}_{h_{u}} \cdot \widetilde{v}_{h_{v}}=\widetilde{u}_{h^{*}}^{*} \cdot \widetilde{v}_{h^{*}}^{*}=\left(\underline{u^{*} \cdot v^{*}}, \overline{u^{*} \cdot v^{*}} ; h^{*}\right),
$$

where:

$$
\begin{aligned}
& \underline{u^{*} \cdot v^{*}}=\min \left\{\underline{u^{*}} \cdot \underline{v}^{*}, \underline{u}^{*} \cdot \bar{v}^{*}, \bar{u}^{*} \cdot \underline{v}^{*}, \bar{u}^{*} \cdot \bar{v}^{*}\right\}, \\
& \overline{u^{*} \cdot v^{*}}=\max \left\{\underline{u^{*}} \cdot \underline{v}^{*}, \underline{u}^{*} \cdot \bar{v}^{*}, \bar{u}^{*} \cdot \underline{v}^{*}, \bar{u}^{*} \cdot \bar{v}^{*}\right\} \text {. }
\end{aligned}
$$


- division

$$
\widetilde{u}_{h_{u}} \div \widetilde{v}_{h_{v}}=\widetilde{u}_{h^{*}}^{*} \div \widetilde{v}_{h^{*}}^{*}=\left(\left(\frac{u^{*}}{v^{*}}\right), \overline{\left(\frac{u^{*}}{v^{*}}\right)} ; h^{*}\right),
$$

where

$$
\begin{aligned}
& \frac{\left(\frac{u^{*}}{v^{*}}\right)}{\overline{u^{*}}}=\min \left\{\underline{u}^{*} / \underline{v}^{*}, \underline{u}^{*} / \bar{v}^{*}, \bar{u}^{*} / \underline{v}^{*}, \bar{u}^{*} / \bar{v}^{*}\right\}, \\
& \frac{u^{*}}{v^{*}}=\max \left\{\underline{u}^{*} / \underline{v}^{*}, \underline{u}^{*} / \bar{v}^{*}, \bar{u}^{*} / \underline{v}^{*}, \bar{u}^{*} / \bar{v}^{*}\right\}
\end{aligned}
$$

and where $\underline{v}_{h_{v}}$ and $\bar{v}_{h_{v}}$ are not zero.

As a straightforward conclusion of the above definition we obtain formulas for crisp semi-numbers. Let $a_{h_{a}}^{\circ}$ and $b_{h_{b}}^{\circ}$ and let $h_{\max }=\max \left\{h_{a}, h_{b}\right\}$. Then we obtain

$$
\begin{aligned}
a_{h_{a}}^{\circ}+b_{h_{b}}^{\circ} & =(a+b)_{h_{\max }}^{\circ}, \\
a_{h_{a}}^{\circ}-b_{h_{b}}^{\circ} & =(a-b)_{h_{\max }}^{\circ}, \\
a_{h_{a}}^{\circ} \cdot b_{h_{b}}^{\circ} & =(a \cdot b)_{h_{\max }}^{\circ}, \\
\frac{a_{h_{a}}^{\circ}}{b_{h_{b}}^{\circ}} & =\left(\frac{a}{b}\right)_{h_{\max }}^{\circ},
\end{aligned}
$$

where $b \neq 0$.

One can easily prove the following lemma.

Lemma 6.1. Trapezoidal equally high fuzzy seminumbers are closed under addition and subtraction operations, i.e.

$$
\widetilde{u}_{h}, \widetilde{v}_{h} \in T F_{h}(\mathbb{R}) \Rightarrow\left(\widetilde{u}_{h}+\widetilde{v}_{h}\right),\left(\widetilde{u}_{h}-\widetilde{v}_{h}\right) \in T F_{h}(\mathbb{R}) .
$$

Lemma 6.2. If $h^{*} \leqslant h$ then $\widetilde{u}_{h}+0_{h^{*}}^{\circ}=\widetilde{u}_{h}$, and $\widetilde{u}_{h} \cdot 1_{h^{*}}^{\circ}=\widetilde{u}_{h}$.

Proof. If $h^{*} \leqslant h$ then $\widetilde{u}_{h}+0_{h^{*}}^{\circ}=(\underline{u}+0, \bar{u}+$ $0, h)=(\underline{u}, \bar{u} ; h)=\widetilde{u}_{h}$, and $\widetilde{u}_{h} \cdot 1_{h^{*}}^{\circ}=(\underline{u} \cdot 1, \bar{u} \cdot 1 ; h)=$ $(\underline{u}, \bar{u} ; h)=\widetilde{u}_{h}$.

In general, trapezoidal fuzzy semi-numbers are not closed under addition and subtraction operations, i.e. addition and subtraction of two arbitrary trapezoidal fuzzy semi-numbers with different heights are not trapezoidal fuzzy semi-numbers. Indeed, since the heights of the two given trapezoidal fuzzy semi-numbers can be different, the one with the smaller height must be elevated before addition or subtraction. Therefore, the elevated fuzzy seminumber can not be a trapezoidal fuzzy semi-number.
Hence, their addition and subtraction can not be a trapezoidal fuzzy semi-number.

Similarly, trapezoidal fuzzy semi-numbers are not closed under multiplication and division operations, i.e. for two arbitrary trapezoidal fuzzy seminumbers $\widetilde{u}_{h}, \widetilde{v}_{h} \in T F S(\mathbb{R})$, their multiplication and division are not trapezoidal fuzzy semi-numbers. Indeed, since left and right spreads of a trapezoidal fuzzy semi-number are polynomials of degree one, their multiplication and division do not yield left and right spreads of degree one. Therefore, their multiplication and division are not trapezoidal fuzzy semi-numbers.

Lemma 6.3. Elementary arithmetic operations between an arbitrary fuzzy semi-number and a crisp number gives a fuzzy number.

Proof. Let $\widetilde{u}_{h}=(\underline{u}, \bar{u} ; h)$ be a fuzzy semi-number with height $h \in(0,1]$ and $k \in \mathbb{R}$. Since $k=(k ; 1)$ and $\max \{h, 1\}=1$, we have the following results:

$$
\begin{aligned}
k+\widetilde{u}_{h} & =(k+\underline{u}, k+\bar{u} ; 1)=(k+\underline{u}, k+\bar{u}), \\
k \cdot \widetilde{u}_{h} & =(k \cdot \underline{u}, k \cdot \bar{u} ; 1)=(k \cdot \underline{u}, k \cdot \bar{u}) .
\end{aligned}
$$

It is also easily seen that for any fuzzy seminumber $\widetilde{u}_{h} \in F S(\mathbb{R})$ the following properties hold

$$
\begin{aligned}
0+\widetilde{u}_{h} & \neq \widetilde{u}_{h}, \\
1 \cdot \widetilde{u}_{h} & \neq \widetilde{u}_{h} .
\end{aligned}
$$

To compute an algebraic expression of given fuzzy semi-numbers, the heights associated with these semi-numbers must be equalized. To this end, after selecting the maximum height of these seminumbers, all the fuzzy semi-numbers are elevated to the maximum height by applying the fuzzy elevator operator. Therefore, now we have an algebraic expression consisting of equiheight fuzzy semi-numbers upon which the elementary arithmetic operators (defined in Section 5) can act. According to this, Arithmetic Mean of $n$ fuzzy semi-numbers, $\widetilde{u}_{i}, i=1, \ldots, n$ denoted as $A M$ is defined as follows:

$$
A M=\frac{1}{n} \sum_{i=1}^{n} \tilde{u}_{i} .
$$

Any arbitrary elementary arithmetic operation between two given fuzzy semi-numbers $\widetilde{u}_{h_{u}}, \widetilde{v}_{h_{v}}$ based on the Extension Principle can be computed 
by applying the aforementioned methods (introduced in this section) and then by obtaining the $h_{*^{-}}$ cut of the result where $h_{*}=\min \left\{h_{u}, h_{v}\right\}$. i.e.

$$
\widetilde{u}_{h_{u}} \odot \widetilde{v}_{h_{v}}=\left[\widetilde{u}_{h^{*}}^{*} \odot \widetilde{v}_{h^{*}}^{*}\right]^{h_{*}},
$$

Here, $\odot$ can be any elementary arithmetic operator.

Let us consider some numerical examples illustrating the proposed methodology.

Example 6.1. Let $\widetilde{u}_{\frac{2}{3}}=\left(-3,-1,2,3 ; \frac{2}{3}\right)$ and $\widetilde{v}_{\frac{1}{3}}=$ $\left(1,2,3,5 ; \frac{1}{3}\right)$ be two fuzzy semi-numbers. They are represented in the parametric form as follows

$$
\begin{aligned}
& \widetilde{u}=\left(\underline{u}, \bar{u} ; h_{u}\right)=\left(-3+3 \alpha, 3-\frac{3}{2} \alpha ; \frac{2}{3}\right), \\
& \widetilde{v}=\left(\underline{v}, \bar{v} ; h_{v}\right)=\left(1+3 \alpha, 5-6 \alpha ; \frac{1}{3}\right) .
\end{aligned}
$$

By applying a fuzzy elevator with respect to maximum height $\frac{2}{3}$ we obtain the associated fuzzy seminumbers $\widetilde{u}_{\frac{2}{3}}^{*}$ and $\widetilde{v}_{\frac{2}{3}}^{*}$ with the following spread functions

$$
\begin{aligned}
& \underline{u}^{*}(\alpha)=\left\{\begin{array}{lc}
-3+3 \alpha, & 0 \leqslant \alpha \leqslant \frac{1}{3}, \\
-3+3 \alpha, & \frac{1}{3} \leqslant \alpha \leqslant \frac{2}{3}, \\
0, & \text { otherwise, }
\end{array}\right. \\
& \bar{u}^{*}(\alpha)=\left\{\begin{array}{lc}
3-\frac{3}{2} \alpha, & 0 \leqslant \alpha \leqslant \frac{1}{3}, \\
3-\frac{3}{2} \alpha, & \frac{1}{3} \leqslant \alpha \leqslant \frac{2}{3}, \\
0, & \text { otherwise, }
\end{array}\right.
\end{aligned}
$$

and

$$
\begin{aligned}
& \underline{v}^{*}(\alpha)= \begin{cases}1+3 \alpha, & 0 \leqslant \alpha \leqslant \frac{1}{3}, \\
2, & \frac{1}{3} \leqslant \alpha \leqslant \frac{2}{3}, \\
0, & \text { otherwise, }\end{cases} \\
& \bar{v}^{*}(\alpha)= \begin{cases}5-6 \alpha, & 0 \leqslant \alpha \leqslant \frac{1}{3}, \\
3, & \frac{1}{3} \leqslant \alpha \leqslant \frac{2}{3}, \\
0, & \text { otherwise. }\end{cases}
\end{aligned}
$$

Now we are able to perform some arithmetic operators. In particular, we obtain the following sum

$$
\widetilde{u}_{\frac{2}{3}}+\widetilde{v}_{\frac{1}{3}}=\widetilde{u}_{\frac{2}{3}}^{*}+\widetilde{v}_{\frac{2}{3}}^{*}=\left(\underline{u}^{*}+\underline{v}^{*}, \bar{u}^{*}+\bar{v}^{*} ; \frac{2}{3}\right),
$$

where

$$
\underline{u}^{*}(\alpha)+\underline{v}^{*}(\alpha)= \begin{cases}-2+6 \alpha, & 0 \leqslant \alpha \leqslant \frac{1}{3} \\ -1+3 \alpha, & \frac{1}{3} \leqslant \alpha \leqslant \frac{2}{3} \\ 0, & \text { otherwise }\end{cases}
$$

$$
\bar{u}^{*}(\alpha)+\bar{v}^{*}(\alpha)=\left\{\begin{array}{lr}
8-\frac{15}{2} \alpha, & 0 \leqslant \alpha \leqslant \frac{1}{3}, \\
6-\frac{3}{2} \alpha, & \frac{1}{3} \leqslant \alpha \leqslant \frac{2}{3}, \\
0, & \text { otherwise. }
\end{array}\right.
$$

The difference looks as follows

$$
\widetilde{u}_{\frac{2}{3}}-\widetilde{v}_{\frac{1}{3}}=\widetilde{u}_{\frac{2}{3}}^{*}-\widetilde{v}_{\frac{2}{3}}^{*}=\left(\underline{u}^{*}-\bar{v}^{*}, \bar{u}^{*}-\underline{v}^{*} ; \frac{2}{3}\right),
$$

where

$$
\begin{aligned}
& \underline{u}^{*}(\alpha)-\bar{v}^{*}(\alpha)= \begin{cases}-8+9 \alpha, & 0 \leqslant \alpha \leqslant \frac{1}{3}, \\
-6+3 \alpha, & \frac{1}{3} \leqslant \alpha \leqslant \frac{2}{3}, \\
0, & \text { otherwise, }\end{cases} \\
& \bar{u}^{*}(\alpha)-\underline{v}^{*}(\alpha)= \begin{cases}2-\frac{9}{2} \alpha, & 0 \leqslant \alpha \leqslant \frac{1}{3}, \\
1-\frac{3}{2} \alpha, & \frac{1}{3} \leqslant \alpha \leqslant \frac{2}{3}, \\
0, & \text { otherwise. }\end{cases}
\end{aligned}
$$

Here is the product

$$
\widetilde{u}_{\frac{2}{3}} \cdot \widetilde{v}_{\frac{1}{3}}=\widetilde{u}_{\frac{2}{3}}^{*} \cdot \widetilde{v}_{\frac{2}{3}}^{*}=\left(\underline{u^{*} \cdot v^{*}}, \overline{u^{*} \cdot v^{*}} ; \frac{2}{3}\right),
$$

where

$$
\begin{aligned}
& \underline{u^{*} \cdot v^{*}}(\alpha)= \begin{cases}(3-3 \alpha)(-5+6 \alpha), & 0 \leqslant \alpha \leqslant \frac{1}{3}, \\
-9+9 \alpha, & \frac{1}{3} \leqslant \alpha \leqslant \frac{2}{3}, \\
0, & \text { otherwise, }\end{cases} \\
& \overline{u^{*} \cdot v^{*}}(\alpha)= \begin{cases}\left(3-\frac{3}{2} \alpha\right)(5-6 \alpha), & 0 \leqslant \alpha \leqslant \frac{1}{3}, \\
9-\frac{9}{2} \alpha, & \frac{1}{3} \leqslant \alpha \leqslant \frac{2}{3}, \\
0, & \text { otherwise. }\end{cases}
\end{aligned}
$$

Finally, we obtain the following ratio

$$
\widetilde{u}_{\frac{2}{3}} \div \widetilde{v}_{\frac{1}{3}}=\widetilde{u}_{\frac{2}{3}}^{*} \div \widetilde{v}_{\frac{2}{3}}^{*}=\left(\left(\frac{u^{*}}{v^{*}}\right), \overline{\left(\frac{u^{*}}{v^{*}}\right)} ; \frac{2}{3}\right),
$$

where

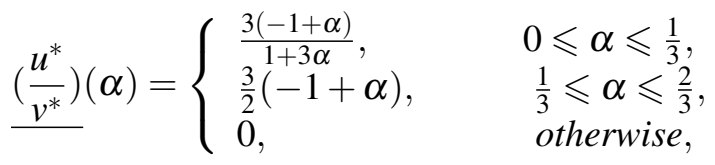

$\overline{\left(\frac{u^{*}}{v^{*}}\right)}(\alpha)= \begin{cases}\frac{6-3 \alpha}{2+6 \alpha}, & 0 \leqslant \alpha \leqslant \frac{1}{3}, \\ -\frac{3}{4}(-2+\alpha), & \frac{1}{3} \leqslant \alpha \leqslant \frac{2}{3}, \\ 0, & \text { otherwise. }\end{cases}$ 
Example 6.2. Let $\widetilde{u}=\left(1,2,3,5 ; \frac{1}{2}\right)$ and $\widetilde{v}=$ $\left(1,3,4,5 ; \frac{1}{3}\right)$ be two fuzzy semi-numbers. The spread functions of $\widetilde{u}, \widetilde{v}$ and the associated elevated fuzzy semi-numbers with respect to the maximum of their heights are shown in Figures 13-16:

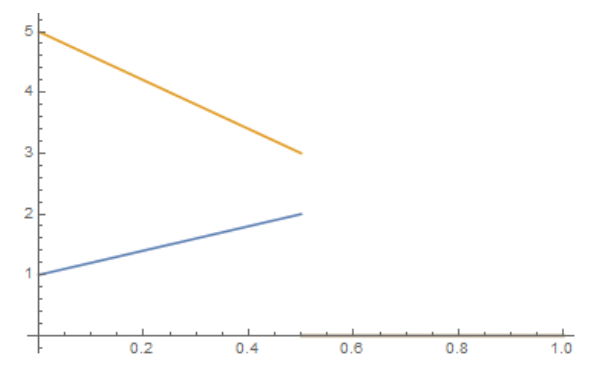

Fig. 13. The spread functions of $\widetilde{u}_{\frac{1}{2}}$.

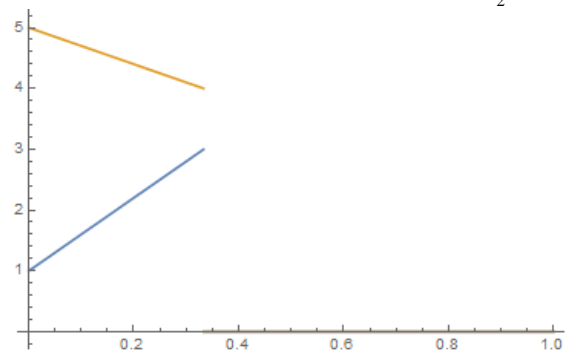

Fig. 14. The spread functions of $\widetilde{v}_{\frac{1}{2}}$.

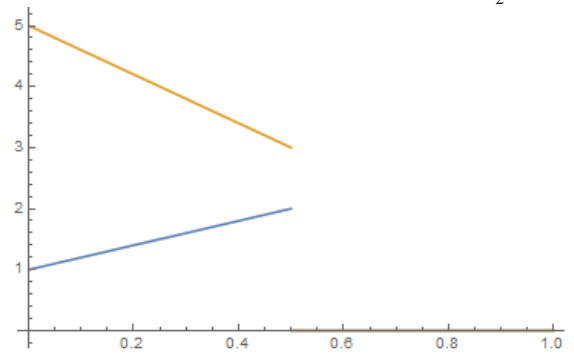

Fig. 15. The spread functions of $\widetilde{u}_{\frac{1}{2}}^{*}$.

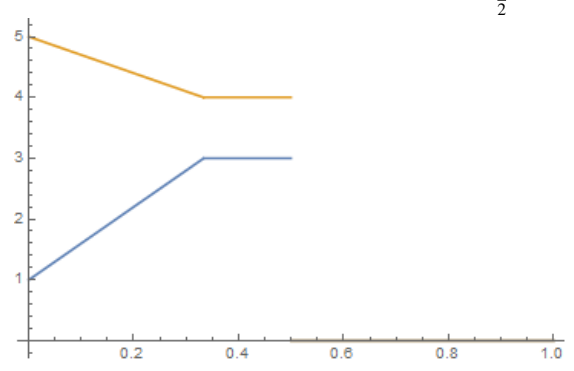

Fig. 16. The spread functions of $\widetilde{v}_{\frac{1}{2}}^{*}$.

The spread functions of the results of addition, subtraction, multiplication and division of the elevated semi-numbers are shown in Figures 17-20

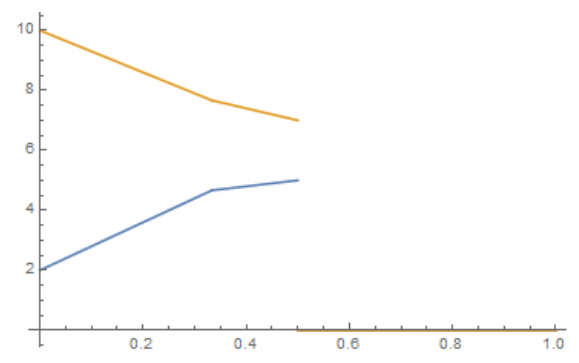

Fig. 17. The spread functions of a sum.

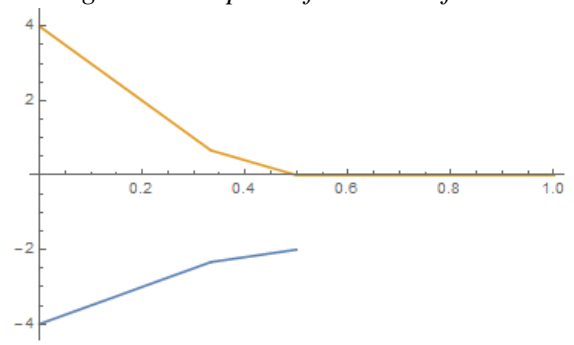

Fig. 18. The spread functions of a difference.

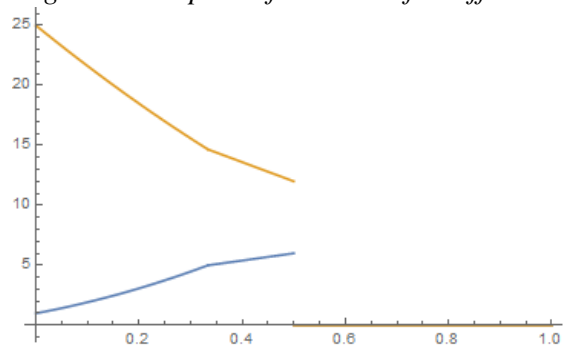

Fig. 19. The spread functions of a product.

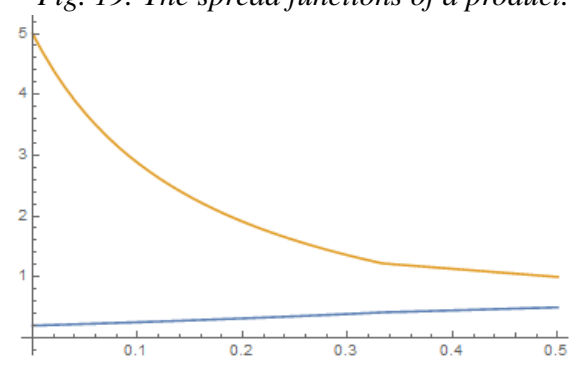

Fig. 20. The spread functions of a ratio.

\section{A medical case study}

Mean Arterial Pressure (MAP), a common term in medicine, indicates the depth of patient's anesthesia 39,40 . This feature helps the anesthetist to monitor patient's fluid balance, ventilation (i.e. the supply of air to the lungs) and drug application. Inhaling anesthetic gases is one of the usual ways to take patients into a deep state of unconsciousness. Isoflurane, as one of these gases, is often deployed in conjunction 
with oxygen or nitrous oxide. In practice the MAP of a patient is measured by a fuzzy logic controller. As a typical example, a surgical team in an operating room uses a MAP controller to know the status of the blood pressure of a patient according to the amount of Isoflurane he has already inhaled. For greater accuracy, the patient blood pressure should be measured (in $\mathrm{mm}$ of $\mathrm{Hg}$ ) at least twice with 2 hours interval. Suppose that the resulting outputs of blood pressure is obtained in terms of two fuzzy sets $\widetilde{B}_{1}, \widetilde{B}_{2}$ with spread functions shown in Figures 21 and 22. These sets express the uncertainty in each blood pressure measurement.

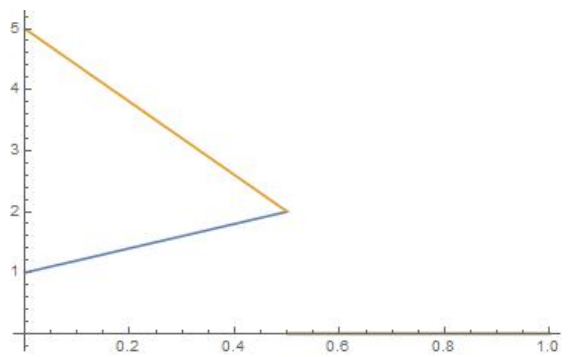

Fig. 21. The spread functions of $\widetilde{B}_{1}$.

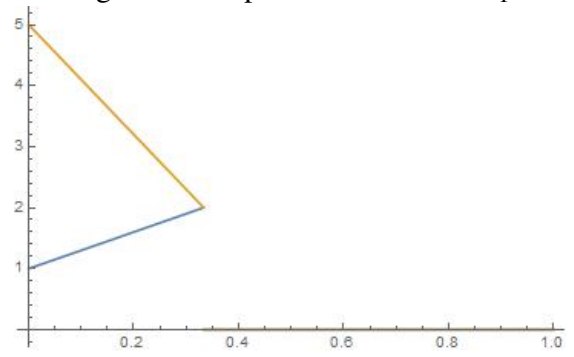

Fig. 22. The spread functions of $\widetilde{B}_{2}$.

An anesthetist needs these two outputs, namely $\widetilde{B}_{1}$ and $\widetilde{B}_{2}$, to be somehow aggregated, so that she/he can make a more prudent and sensible decision about the anesthesic depth of a patient. The fundamental part of such aggregations is often done via normalization which is the most prevalent and somewhat even the sole way today. One approach to aggregating $\widetilde{B}_{1}$ and $\widetilde{B}_{2}$ is to take their arithmetic mean.

A traditional way for calculating the arithmetic mean of non-normal fuzzy sets goes through their normalization. However, in our case, $\widetilde{B}_{1}$ and $\widetilde{B}_{2}$ after normalization are indistinguishable (see Figures 23 and 24) co, consequently, the arithmetic mean of their normalized forms results in just the same fuzzy set.

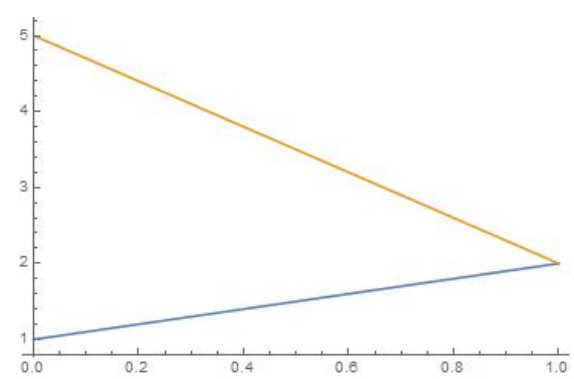

Fig. 23. The spread functions of $\widetilde{B}_{1}$ after normalization.

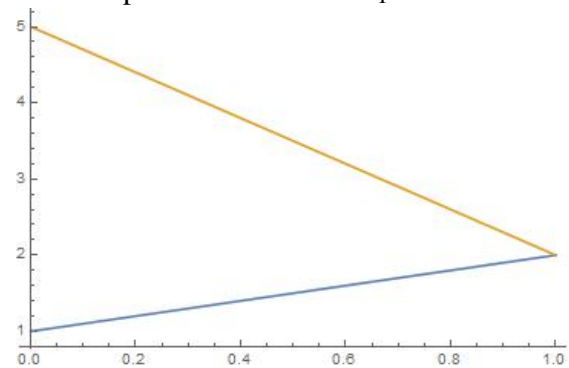

Fig. 24. The spread functions of $\widetilde{B}_{2}$ after normalization.

However, by applying our elevation method for computing the arithmetic mean of $\widetilde{B}_{1}$ and $\widetilde{B}_{2}$ we get two equally high fuzzy sets while at the same time their differences are preserved. Figures 25, 26 and 27 illustrate these elevated values and their associated mean value, respectively.

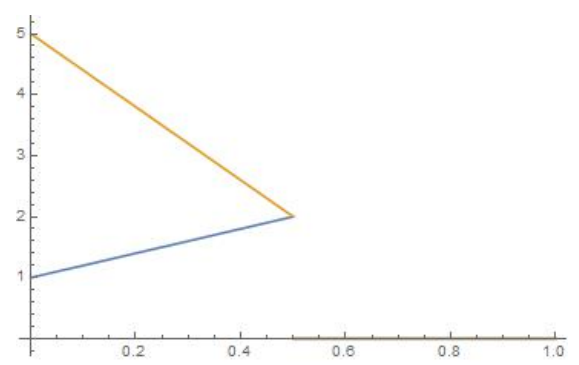

Fig. 25. The spread functions of $\widetilde{B}_{1}$ in elevated form. 


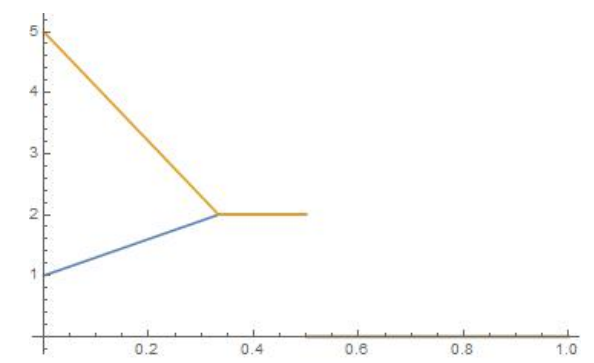

Fig. 26. The spread functions of $\widetilde{B}_{2}$ in elevated form.

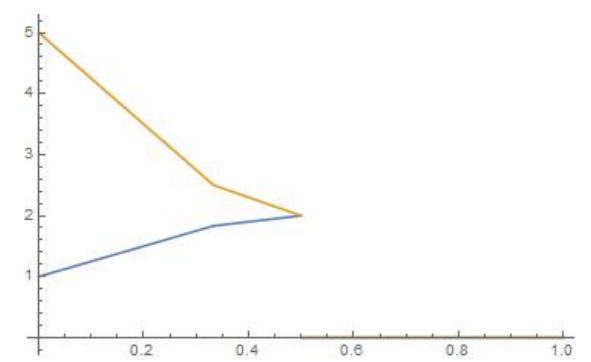

Fig. 27. The spread functions of the arithmetic mean of elevated $\widetilde{B}_{1}$ and $\widetilde{B}_{2}$.

As it has been shown, the normalization approach may lead to misleading results. Indeed, through the deformation of the shape of the input data caused by the normalization process we have lost some information on the initial differences between fuzzy observations. Fortunately, the proposed elevation method removes this weakness. It is obvious that such information loss may cause incorrect or false conclusions which sometimes may can result in serious and irrecoverable medical faults.

\section{Conclusions}

Fuzzy numbers of height one has formed the cornerstone of normal fuzzy sets. Based on this assumption, not only rudimentary arithmetic has been established but also, other topics e.g. fuzzy ranking, fuzzy approximation, fuzzy differential equations, fuzzy integral equations and systems of fuzzy equations have been developed.

Having gone against this prevailing thought of height one in fuzzy numbers, in this paper, we considered fuzzy sets not to be restricted by any predefined height. To achieve this goal we introduced the concept of fuzzy semi-number. Next we investigated elementary arithmetic on equally high fuzzy seminumbers. Then a new method, based on elevation, for defining algebraic operations on general fuzzy semi-numbers in general form was introduced. As it was demonstrated in medical case study and some numerical examples, using the proposed methodology we avoid a loss of information in processing fuzzy data which may be caused by the traditional normalization method.

\section{References}

1. S. Abbasbandy, B. Asady, The nearest trapezoidal fuzzy number to a fuzzy quantity, Journal of Applied Mathematics and Computations, 156 (2004) 381-386.

2. S. Abbasbandy, M. Amirfakhrian, The nearest approximation of a fuzzy quantity in parametric form, Journal of Applied Mathematics and Computations, 172 (2006) 624-632.

3. S. Abbasbandy, M. Amirfakhrian, The nearest trapezoidal form of a generalized left right fuzzy number, Journal of Approximate Reasoning, 43 (2006) 166178.

4. M. Amirfakhrian, Properties of parametric form approximation operator of fuzzy numbers, Analele Stiintifice ale Universitatii Ovidius Constanta, 18 (2010) 23-34.

5. M. Amirfakhrian, Sh. Yeganehmanesh, P. Grzegorzewski, A new distance on fuzzy semi-numbers, Soft Computing, (2017) https://doi.org/10.1007/s00500-017-2902-2.

6. L. Anzilli, G. Facchinetti, G. Mastroleo, A parametric approach to evaluate fuzzy quantities, Fuzzy Sets and Systems, 250 (2014) 110-133.

7. A. I. Ban, L. C. Coroianu, Simplifying the Search for Effective Ranking of Fuzzy Numbers, IEEE Transactions on Fuzzy Systems, 23(2) (2015) 327-339.

8. A. I. Ban, L. Coroianu, A. Khastan, Conditioned weighted LR approximations of fuzzy numbers, Fuzzy Sets and Systems, 62 (2015) 1-26.

9. A. I. Ban, L. Coroianu, P. Grzegorzewski, Trapezoidal approximation and aggregation, Fuzzy Sets and Systems, 177(1) (2011) 45-59.

10. S. Banerjee, Arithmetic Operations on Generalized Trapezoidal Fuzzy Number and its Applications, Turkish Journal of Fuzzy Systems, 3(1) (2012) 16-44.

11. D. Behera, S. Chakraverty, Solving fuzzy complex system of linear equations, Informations Science, 277 (2014) 154-162.

12. D. Chakraborty, D. Guha, Addition of two Generalized Fuzzy Numbers, International Journal of Industrial Mathematics, 2(1) (2010) 9-20.

13. S. Chanas, On the interval approximation of a fuzzy 
number, Fuzzy Sets and Systems, 122 (2001) 353-356.

14. P.T. Chang, E.S. Lee, Ranking of fuzzy sets based on the concept of existence, Computers and Mathematics with Applications, 27(10) (1994) 1-21.

15. S. H. Chen, Ranking fuzzy numbers with maximizing set and minimizing set, Fuzzy Sets and Systems, 17(2) (1985) 113-129.

16. L. Coroianu, M. Gagolewski, P. Grzegorzewski, Nearest piecewise linear approximation of fuzzy numbers, Fuzzy Sets and Systems, 233 (2013) 26-51.

17. M. Delgado, M. A. Vila, W. Voxman, On a canonical representation of fuzzy numbers, Fuzzy Sets and Systems, 93 (1998) 125-135.

18. S. L. Dong, H. Vu, D .N. Phu, The formulas of the solution for linear-order random fuzzy differential equations. Journal of Intelligent and Fuzzy Systems, 28(2) (2015) 795-807.

19. D. Dubois and H. Prade, Operations on fuzzy numbers, International Journal Systems Science, 9 (1978) 613-626.

20. D. Dubois, H. Prade, Fuzzy Sets and Systems: Theory and Application, Academic Press, New York, (1980).

21. M. A. Fariborzi Araghi, Gh. Kazemi Gelian, Solving fuzzy Fredholm linear integral equations using Sinc method and double exponential transformation, Soft Computing, 19(4) (2015) 1063-1070.

22. L. S. Gao, The fuzzy arithmetic mean, Fuzzy Sets and Systems, 107(3) (1999) 335-348.

23. P. Grzegorzewski, Nearest interval approximation of a fuzzy number, Fuzzy Sets and Systems, 130 (2002) 321-330.

24. P. Grzegorzewski, E. Mrówka, Trapezoidal approximations of fuzzy numbers, Fuzzy Sets and Systems, IFSA 2003, T. Bilgic, B. DeBaets, O. Kay nak (Eds.), Lecture Notes in Artificial Intelligence 2715, Springer, (2003) 237-244.

25. P. Grzegorzewski, E. Mrówka, Trapezoidal approximations of fuzzy numbers, Fuzzy Sets and Systems, 153 (2005) 115-135.

26. D. Guha, D. Chakraborty, A new approach to fuzzy distance measure and similarity measure between two generalized fuzzy numbers, Applied Soft Computing, 10 (2010) 90-99.

27. A. Khastan, R. Rodriguez-López, On periodic solutions to first order linear fuzzy differential equations under differential inclusions' approach, Information
Science, 322 (2015) 31-50.

28. M. Ma, M. Friedman, A. Kandel, A new fuzzy arithmetic, Fuzzy Sets and Systems, 108 (1999) 83-90.

29. M. Ma and A. Kandel and M. Friedman, A new approach for defuzzification, Fuzzy Sets and Systems, 111 (2000) 351-356.

30. M. Ma and A. Kandel and M. Friedman, Correction to "A new approach for defuzzification", Fuzzy Sets and Systems, 128 (2002) 133-134.

31. V. H. Ngo, Fuzzy fractional functional integral and differential equations, Fuzzy Sets and Systems, 280 (2015) 58-90.

32. S. Pushpinder, A novel method for ranking generalized fuzzy numbers, Information Science and Engineering, 31 (2015) 1373-1385.

33. X. Qu, F. Sun, T. Wang, Matrix elementary transformations in solving systems of fuzzy relation equations, Applied Soft Computing, 31 (2015) 25-29.

34. W. Voxman, Some remarks on distance between fuzzy numbers, Fuzzy Sets and Systems, 100 (1998) 353365.

35. R. R. Yager, On the normalization of fuzzy belief structures, International Journal of Approximate Reasoning, 14(2) (1996) 127-153.

36. J. S. Yao, K. Wu, Ranking fuzzy numbers based on decomposition principle and signed distance, Fuzzy Sets and Systems, 116 (2000) 275-288.

37. Sh. Yeganehmanesh, M. Amirfakhrian, An Approximation of Fuzzy Numbers Based on Polynomial Form Fuzzy Numbers, International Journal of Analysis and Applications, 16(2) (2018) 290-305.

38. Sh. Yeganehmanesh, M. Amirfakhrian, P. Grzegorzewski , Fuzzy semi-numbers and a distance on them with a case study in medicine, Mathematical Sciences, 12(1)(2018) 4152.

39. H. Ying, L.C. Sheppard, Regulating mean arterial pressure in postsurgical cardiac patients, IEEE Engineering in Medicine and Biology Magazine, 13(5) (1994) 671-677.

40. A.M. Zbinden, P. Feigenwinter, S. Petersen-felix and S. Hacisalizade, Arterial pressure control with isoflurane using fuzzy logic, British Journal of Anaesthesia, 74 (1995) 66-72.

41. H.J. Zimmermann, Fuzzy Set Theory and Its Applications, $2^{\text {nd }}$ Edition, Kluwer Academic, Boston, (1991). 